\title{
Robust Loss Filter Design for Digital Waveguide Synthesis of String Tones
}

\author{
Balázs Bank and Vesa Välimäki, Senior Member, IEEE
}

\begin{abstract}
A robust loss filter design method is presented for digital waveguide string models, which can be used with high filter orders. The method aims at minimizing the decay time error in partials of the synthetic tone. This is achieved by a new weighting function based on the first-order Taylor series approximation of the decay time errors. Smoothing of decay time data and requiring the design to be minimum-phase are also proposed to facilitate the stability of the design. The new method is applicable to analysisbased sound synthesis of piano and guitar tones, for example.
\end{abstract}

Index Terms-Acoustic signal processing, digital filter design, electronic music, music synthesis, musical instruments.

\section{INTRODUCTION}

$\mathbf{P}$ HYSICAL MODELING of musical instruments using digital waveguides [1] has been an active field over the past decade. This letter describes a robust technique for high-order loss filter design for digital waveguide models of string instruments. Previous methods have been useful in designing firstorder loss filters but have turned out to be imperfect in the case of higher filter orders. The proposed method optimizes for decay times, which was found to be a perceptually meaningful criterion.

Section II of this letter describes the basic idea of digital waveguide synthesis. The commonly used technique for loss filter design is presented in Section III. A new design method is proposed in Section IV based on a special weighting function, and Section $\mathrm{V}$ discusses the phase specification for loss filter design. The properties of the new method are demonstrated in the case of weighted least squares IIR filter design in Section VI. Finally, conclusions are given in Section VII.

\section{Digital WaVeguide StRing Model}

The digital waveguide [1] originates from the discretization of the traveling-wave solution of the wave equation. By assuming linearity, all the losses and dispersion of the string and

\footnotetext{
Manuscript received December 19, 2000; revised July 15, 2002. This work was supported by the Academy of Finland, within the framework of the Sound Source Modeling Project. The work of V. Välimäki was supported by a postdoctoral research grant from the Academy of Finland and was performed in 2000 while at the Helsinki University of Technology before joining the Pori School of Technology and Economics.

B. Bank was with Helsinki University of Technology, Laboratory of Acoustics and Audio Signal Processing. He is now with Budapest University of Technology and Economics, Department of Measurement and Information Systems, H-1521 Budapest, Hungary (e-mail: bank@mit.bme.hu).

V. Välimäki was with the Pori School of Technology and Economics, Tampere University of Technology. He is now with Helsinki University of Technology, Laboratory of Acoustics and Audio Signal Processing, FIN-02015 HUT, Espoo, Finland (e-mail: vesa.valimaki@hut.fi).

Digital Object Identifier 10.1109/LSP.2002.806707
}

the terminations can be lumped to one point of the model [1]. Thus, the model reduces to a delay line and a filter in a feedback loop [2], [3].

Such a model has the transfer function of $1 /\left(1-z^{-L} H(z)\right)$ where $L$ is the length of the delay line in samples, and $H(z)$ is the loop filter. This model is capable to generate a quasi-harmonic tone consisting of exponentially decaying sinusoids. The partial frequencies are determined by the delay line length $L$ and the phase delay of the filter $H(z)$. The decay times of the partials are controlled by the magnitude frequency response of the loop filter $H(z)$.

In [4], two sophisticated filter design techniques presented in [5] are discussed, which design the loop filter $H(z)$ as a whole. The results show that these complicated methods usually result in filter magnitudes larger than one, i.e., in unstable feedback loops. Stable loop filters have been obtained by neural-network-based optimization [6]. However, the required order of the filters are of a magnitude larger than we are interested in this study. In general, designing $H(z)$ is problematic because different accuracy is required with respect to the magnitude and phase response of the filter.

To simplify the design, the filter $H(z)$ is usually factored to three parts: $H(z)=H_{l}(z) H_{d}(z) H_{f d}(z)$, where $H_{l}(z)$ is responsible for the losses and $H_{d}(z)$ for the dispersion. The fractional delay filter $H_{f d}(z)$ is used for fine-tuning the fundamental frequency of the tone. A further advantage of this approach is that the string parameters, such as the length or losses due to the touch of the finger, can be changed separately during playing. Here we concentrate on designing the loss filter $H_{l}(z)$.

\section{LOSS FILTER DESIGN}

In digital waveguide synthesis, the goal is usually to produce synthetic sounds similar to real instruments. In the case of string instruments, a robust analysis method consists in measuring the decay times of the partials of a recorded single tone [3]. From the decay times, the required loop gain for each partial frequency can be calculated by $g_{k}=e^{-1 /\left(f_{0} \tau_{k}\right)}$ where $f_{0}$ is the fundamental frequency of the string, and $\tau_{k}$ is the decay time constant of the $k$ th partial. The loop gain values $g_{k}$ together with the partial frequencies $\vartheta_{k}$ constitute the magnitude specification for the filter $H_{l}(z)$, i.e., $\left|H_{l}\left(e^{j \vartheta_{k}}\right)\right|=g_{k}$.

A simple and common technique for designing the filter $H_{l}(z)$ is minimizing the mean-squared error $\sum_{k=1}^{K}\left(\left|H_{l}\left(e^{j \vartheta_{k}}\right)\right|-g_{k}\right)^{2}$, where $K$ refers to the number of measured partials. Nevertheless, problems arise because the decay times are a nonlinear function of the filter magnitude response, namely $\tau_{k}=-1 /\left(f_{0} \ln g_{k}\right)$. Therefore, as $g_{k}$ approaches unity, the same amount of magnitude deviation 
$\hat{g}_{k}-g_{k}$ will correspond to a larger and larger difference in the decay time $\hat{\tau}_{k}-\tau_{k}$. Moreover, if the magnitude response of $H_{l}(z)$ exceeds unity at one of the partial frequencies, the digital waveguide becomes unstable, since the loop gain is larger than one.

In [3], an ad hoc weighting function $w_{k}=1 /\left(1-g_{k}\right)$ for the one-pole loop filter is proposed, which helps to lessen these problems. A robust method designing the one-pole loss filter was presented in [7, pp. 87-91]. Bank [7, pp. 82-87] presented a method for high-order loss filter design based on the transformation of the specification. A two-step procedure for high-order loss filter design applying polynomial fitting was proposed in [8]. In the following, a simple and robust method is presented, which can be used with any filter order and with different filter design algorithms.

\section{NEW DESIGN METHOD}

The role of the loss filter is to set the decay times. Therefore, it is reasonable to minimize the error with respect to the time constants, which was found to be a perceptually appropriate criterion. For example, in the mean-squares sense, the error is $e_{\tau}=\sum_{k=1}^{K}\left(\hat{\tau}_{k}-\tau_{k}\right)^{2}$ where $\tau_{k}$ are the prescribed and $\hat{\tau}_{k}$ are the approximated decay times. The decay times of the synthetic tone can be computed from the magnitude of the designed filter $\hat{g}_{k}=\left|H_{l}\left(e^{j \vartheta_{k}}\right)\right|$ by $\hat{\tau}_{k}=f\left(\hat{g}_{k}\right)=-1 /\left(f_{0} \ln \hat{g}_{k}\right)$. If the function $f\left(\hat{g}_{k}\right)$ is approximated by the first-order Taylor polynomial around the specification $g_{k}$, we obtain

$$
\begin{aligned}
e_{\tau} & =\sum_{k=1}^{K}\left(f\left(\hat{g}_{k}\right)-f\left(g_{k}\right)\right)^{2} \approx \sum_{k=1}^{K}\left(f^{\prime}\left(g_{k}\right)\left(\hat{g}_{k}-g_{k}\right)\right)^{2} \\
& =\sum_{k=1}^{K} w_{k}\left(\hat{g}_{k}-g_{k}\right)^{2}
\end{aligned}
$$

which is a simple mean-squares minimization with weights $w_{k}=\left(f^{\prime}\left(g_{k}\right)\right)^{2}$, where $f^{\prime}\left(g_{k}\right)$ is the differential of the function $f$ at the specified magnitude $g_{k}$. Similar derivations can be performed for other error criteria (e.g., minimax). Note that now the weights depend on the magnitude specification and not on the frequencies, which is more common in digital filter design.

The first derivate of $f\left(g_{k}\right)$ is $f^{\prime}\left(g_{k}\right)=1 /\left(f_{0} g_{k}\left(\ln g_{k}\right)^{2}\right)$. For $g_{k}=1-\epsilon, 0<\epsilon \ll 1$, which is generally the case, $f^{\prime}\left(g_{k}\right)$ can be approximated by $f^{\prime}\left(g_{k}\right) \approx 1 /\left(f_{0}\left(g_{k}-1\right)^{2}\right)$. This comes from the first-order Taylor series approximation of $\ln g_{k}$. Since $1 / f_{0}$ does not depend on $k$, it can be omitted from the weighting function. Hence, the weighting function becomes

$$
w_{k}=\frac{1}{g_{k}^{2}\left(\ln g_{k}\right)^{4}} \approx \frac{1}{\left(g_{k}-1\right)^{4}} .
$$

A similar weighting function based on the time-constant error has been derived for the $L^{\infty}$ norm in [5, pp. 182-183].

The approximation of (1) is accurate only for $\hat{g}_{k} \approx g_{k}$, which means that the magnitude of the designed filter is close to the specification. Caused by several factors, the measured decay times have a great variance [8], which cannot be followed by filters of reasonable order $(N<20)$. Therefore, it is worthwhile to smooth the decay time data $\tau_{k}$, e.g., by convolving them with a short window function before computing the specification $g_{k}$. This way, the condition $\hat{g}_{k} \approx g_{k}$ can be assured.

\section{PHASE}

The magnitude specification $g_{k}$ and the weights $w_{k}$ can be directly used for linear-phase finite-impulse response filter design. However, by doing so, half of the degrees of freedom are wasted for demanding the impulse response to be symmetric. In practice, it is not necessary to have an exactly linear-phase loss filter, since a nonlinear phase response corresponds to a slightly inharmonic tone, which does not corrupt the sound quality.

Designing minimum-phase filters is a pleasant choice, since then the phase specification can be easily computed from the logarithm of the magnitude specification by Hilbert transform [9]. Note that the Hilbert transform needs magnitude data for the entire digital frequency band and on a linear frequency scale. The missing data points in the high-frequency region are proposed to be calculated by designing a one-pole filter for the specification, e.g., by one of the methods presented in [7, pp. 87-91] or in [3]. Then, the magnitude response of the one-pole filter is used as a specification for the high frequencies. This is reasonable, since the loss filter behavior in the high-frequency region has no significant influence on the resulted tone, and such a simple specification is easily fulfilled by the filter design. At the original data points of the highest specified frequencies, a crossfade is applied to avoid discontinuities.

\section{DESIGN EXAMPLES}

Examples are presented for IIR filter design. For the examples, the weighted least squares method implemented in MATLAB's invfreqz function is used [10]. The decay time data are smoothed by convolving them with a triangular window $[0.25,0.5,0.25]$. Here, the last five data points of the measured specification are linearly mixed to the magnitude response of the designed one-pole filter. The magnitude response on a dense linear grid is calculated by using third-order polynomial interpolation, which was found to be accurate enough. The phase response on this dense grid is computed by the Hilbert transform and then resampled at the frequencies of the original specification points.

The decay time data used for this example was calculated from a piano tone $F_{2}^{\sharp}\left(f_{0}=92.2 \mathrm{~Hz}\right)$, near-field recording. The decay rate of the partials up to $6.57 \mathrm{kHz}$ were measured, which yielded data for 64 partials. The sampling frequency is $f_{s}=22.05 \mathrm{kHz}$. The smoothed decay times are displayed with points in Fig. 1(c). The filter magnitude specification calculated from the smoothed decay times has been plotted with points in Fig. 1(a) and (b).

IIR filters of order 2, 8 , and 16 were designed. The magnitude responses are depicted in Fig. 1(a), and Fig. 1(b) shows the same curves magnified for the most relevant frequency and magnitude region. Fig. 1(c) shows the corresponding decay times.

Fig. 1 reveals that the magnitude error is smaller where the specification $g_{k}$ is closer to unity, which is necessary for the equal accuracy in decay times. Similar results have been obtained with several cases of piano and guitar data. The magnitude response of the designed filters never exceeded unity, i.e., 

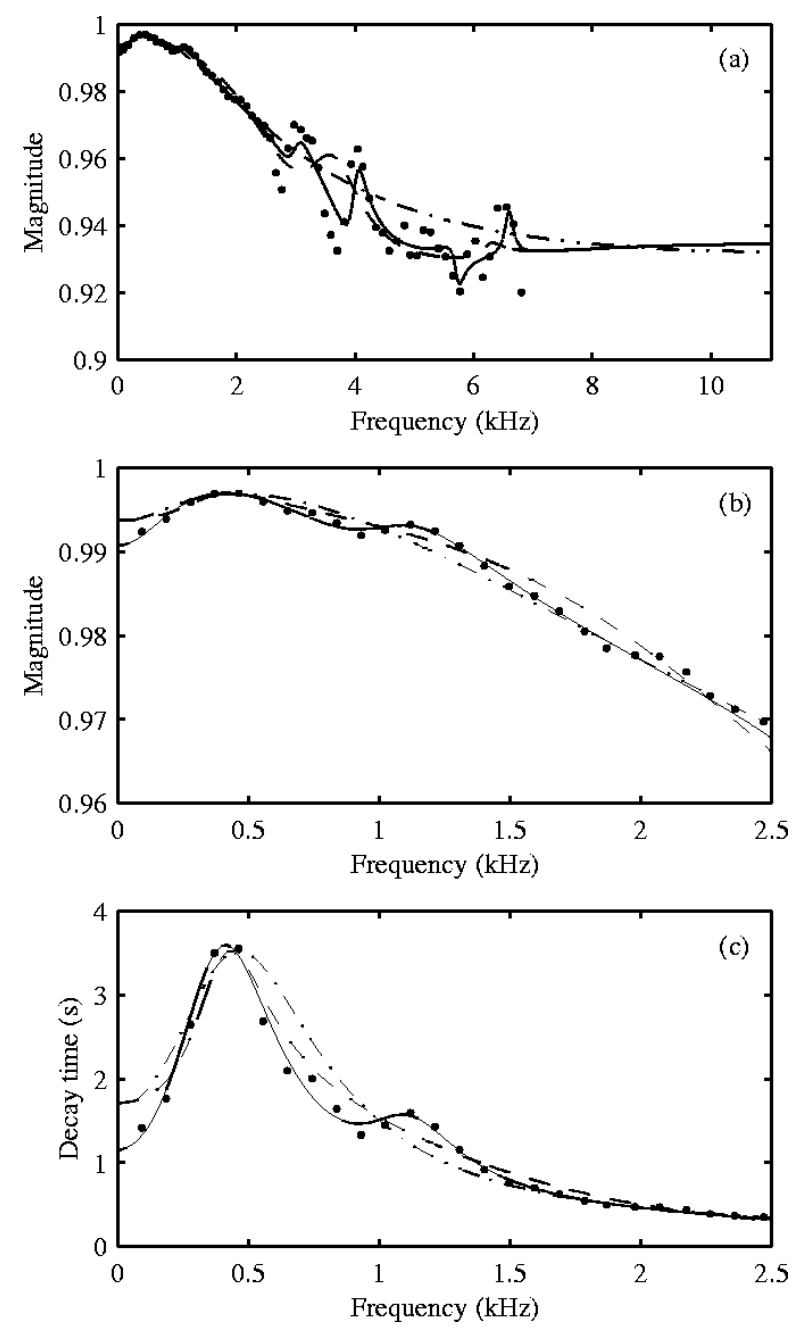

Fig. 1. IIR loss filter magnitude responses (a) for the full frequency band and (b) at low frequencies up to $2.5 \mathrm{kHz}$, and the corresponding decay times (c) for filter orders of two (dashed-dotted line), 8 (dashed line), and 16 (solid line). The specification is depicted with dots in each case.

the digital waveguide loop remained always stable. Proper listening tests should be conducted to find out the sufficient filter order that enables natural-sounding resynthesis.

\section{CONCLUSION}

A new method was presented that is targeting to optimize the decay times of the digital waveguide and that can be used for high filter orders. The weighting is based on the first-order Taylor series approximation of the decay time errors. Smoothing of target decay time specification is necessary in the case of high variance of measured decay times. Requiring the design to be minimum-phase is also proposed. Examples of weighted least squares IIR loss filter design were shown. The new method is useful in analysis-based synthesis of string instrument sounds, such as piano and guitar tones.

\section{ACKNOWLEDGMENT}

The authors would like to thank C. Erkut, I. Kollár, and L. Sujbert for their helpful comments.

\section{REFERENCES}

[1] J. O. Smith, "Physical modeling using digital waveguides," Comput. Music J., vol. 16, no. 4, pp. 74-91, Winter 1992.

[2] D. A. Jaffe and J. O. Smith, "Extensions of the Karplus-Strong pluckedstring algorithm," Comput. Music J., vol. 7, no. 2, pp. 56-69, 1983.

[3] V. Välimäki, J. Huopaniemi, M. Karjalainen, and Z. Jánosy, "Physical modeling of plucked string instruments with application to real-time sound synthesis," J. Audio Eng. Soc., vol. 44, no. 5, pp. 331-353, May 1996.

[4] J. Laroche and J.-M. Jot, "Analysis/synthesis of quasiharmonic sounds by use of the Karplus-Strong algorithm," in Proc. 2nd French Congress Acoustics, Apr. 1992.

[5] J. O. Smith, "Techniques for digital filter design and system identification with application to the violin," Ph.D. dissertation, Stanford Univ., Stanford, CA, June 1983.

[6] A. W. Y. Su and S.-F. Liang, "A new automatic IIR analysis/synthesis technique for plucked string instruments," IEEE Trans. Speech Audio Processing, vol. 9, pp. 747-754, July 2001.

[7] B. Bank, "Physics-based sound synthesis of the piano," Master's thesis, Budapest Univ. Technol. and Economics, Budapest, Hungary, May 2000. Published as Rep. 54 of Helsinki Univ. of Technol., Lab. of Acoustics and Audio Signal Processing. [Online] Available: http://www.mit.bme.hu/ bank.

[8] C. Erkut, "Model order selection techniques for the loop filter design of virtual instruments," in Proc. 5th Conf. Systemics, Cybernetics and Informatics, vol. 10, Orlando, FL, July 2001, pp. 529-534.

[9] A. V. Oppenheim and R. W. Schafer, Digital Signal Processing. Englewood Cliffs, NJ: Prentice-Hall, 1975.

[10] Matlab 5 Manual. Natick, MA: Mathworks, 1996. 